\title{
Abdominal wall desmoid tumor mimicking a subserosal uterine leiomyoma
}

This article was published in the following Dove Press journal:

International Journal of General Medicine

7 June 2011

Number of times this article has been viewed

\section{Moamar Al-Jefout ${ }^{1,2}$ \\ Alabed Walid ${ }^{2}$ \\ Abomayale Esam² \\ Alqaisi Amin ${ }^{2}$ \\ Hawa Nather ${ }^{1,2}$ \\ Nawayse Sultan ${ }^{2}$ \\ Khadra Maysa ${ }^{3}$}

'Department of Obstetrics and Gynaecology, Mutah Medical Faculty, Mutah University, Karak; ${ }^{2}$ Karak Hospital, Ministry of Health, Karak;

${ }^{3}$ University of Jordan, Amman, Jordan
Correspondence: Moamar Al-Jefout Department of Obstetrics and Gynaecology, Mutah Medical Faculty, Mutah University, Karak, Jordan Tel +962797377790

Fax +96232375540

Email drmoamar@yahoo.co.uk
Abstract: Desmoid tumors are cytologically bland fibrous neoplasms originating from musculoaponeurotic structures throughout the body. The cause of desmoid tumors is uncertain, but may be related to trauma or hormonal factors, or may have a genetic association. These tumors can be found in some young women during pregnancy or just after giving birth. We report herein a case of desmoid tumor on the inner aspect of the abdominal wall that mimicked a large subserosal uterine leiomyoma. Initial clinical examination of the patient suggested a large abdominal wall tumor, while the imaging techniques including transabdominal ultrasound and magnetic resonance imaging suggested a large subserosal uterine leiomyoma as the initial diagnosis. This case emphasizes the importance of clinical examination during the diagnostic process.

Keywords: diagnosis, lesion, ultrasound

\section{Introduction}

Desmoid tumors are cytologically bland fibrous neoplasms originating from musculoaponeurotic structures throughout the body. The term "desmoid", coined by Muller in 1838 , is derived from the Greek word "desmos", which means tendonlike. Overall, desmoid tumors are reported to account for $0.03 \%$ of all neoplasms. ${ }^{1}$ When present in patients with familial polyposis of the colon, the prevalence of desmoid tumors is as high as $13 \% .{ }^{2}$ In females, desmoid tumors are usually found in the reproductive age group and may appear just after giving birth.

Desmoid tumors may be extra-abdominal (typically in the shoulder, chest wall, thigh, and back), abdominal (typically in aponeurotic structures of the abdominal wall), or intra-abdominal (typically in the mesentery and pelvic walls). At gross examination, these masses are typically firm and nonencapsulated, with coarse white trabeculae that simulate scar tissue.

Desmoid tumors are usually well differentiated and appear as infiltrative solid overgrowths of fibrous tissue. At histologic analysis, desmoid tumors consist of elongated spindle-shaped cells separated by dense bands of collagen. Moreover, they are locally aggressive, and tend to recur, making treatment of these relatively rare fibrous tumors challenging. When surgical excision is not complete, they tend to recur. Therefore, gross total resection with a $3 \mathrm{~cm}$ safe margin is the indicated initial therapy, if it can be performed without significant disfigurement. ${ }^{3-6}$ In addition to surgery, radiotherapy is also excellent for obtaining local control, even in patients with a large burden of recurrent disease. Radiation doses in the range of 50-55 Gy give a chance of local control equal to that obtained with higher doses. ${ }^{3}$ 
A meticulous clinical examination supported by ultrasound imaging can be a very useful tool diagnosis of these tumors. ${ }^{7}$ A typical sonographic feature of all intramuscular desmoids is the spindle-shaped tumor margins when scanning along the long axis of the affected muscle. However, desmoids arising from the fascia have an irregular shape with rich vascularity. ${ }^{8}$ These tumors are hypointense on T1-weighted MRI and have variable signal intensity on T2-weighted images. ${ }^{9}$ In addition, computed tomographic scanning can also be used for diagnosis, because these tumors are usually located superficially. ${ }^{10}$

\section{Case report}

A 34-year-old multiparous Jordanian woman attended the gynecological outpatient clinic at Al-Karak Hospital, a small peripheral hospital in the southern part of Jordan. She was complaining of lower abdominal swelling. The swelling was noticed initially during her last pregnancy 4 months earlier, and rapidly enlarged after giving birth. On initial clinical examination, the swelling was located in the right iliac fossa as a firm mass measuring around $8 \times 8 \mathrm{~cm}$ with limited mobility and not attached to the skin. Bimanual pelvic examination revealed a normal-sized uterus free from any attached masses and with no adnexal masses. An abdominal wall tumor was suspected. However, to be sure, abdominal ultrasound and MRI were performed. The initial transabdominal scan (Figure 1) suggested a large subserosal leiomyoma. The MRI imaging report also suggested a subserosal tumor as the first diagnosis (Figure 2). The MRI without and with intravenous Gd-DTPA: all pulse sequences were obtained (GE 16 channels, 1.5 Tesla) reported a large, well-defined, oval-shaped, abdominopelvic mass lesion, measuring about $12 \times 8 \mathrm{~cm}$ and overlapping the right side of the pelvic cavity anteriorly. The lesion has an

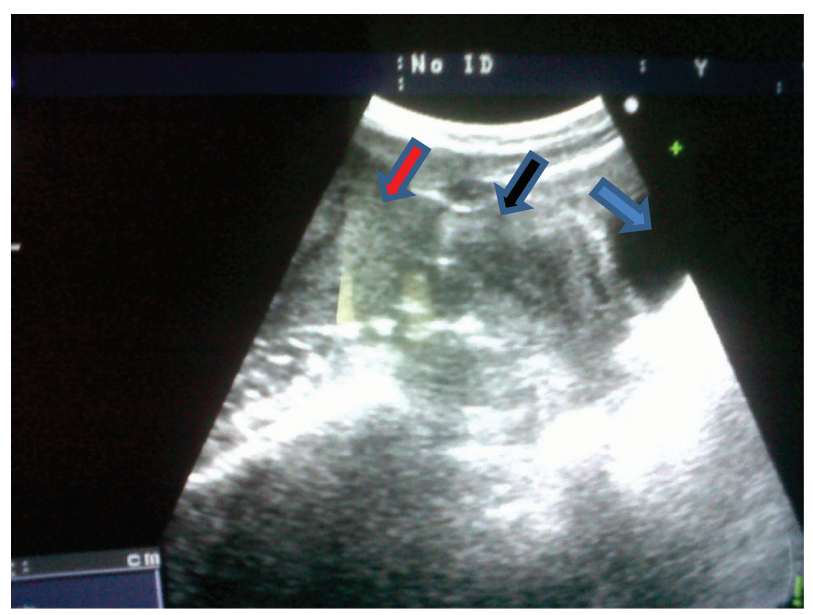

Figure I A transabdominal ultrasound scan showing the mass (red arrow) and the uterus (black arrow), bladder (blue arrow).

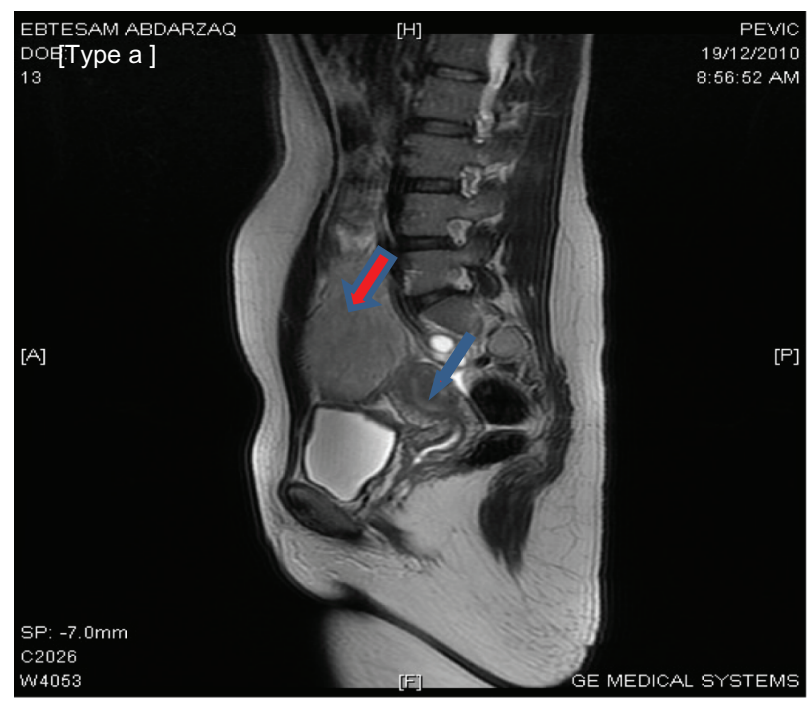

Figure 2 Pelvi-abdominal MRI showing a large, well-defined, oval mass measuring $12 \times 8 \mathrm{~cm}$ in dimensions overlapping the right side of the pelvic cavity (red arrow). The mass appears to be connected to the uterus (blue arrow). A subserosal leiomyoma is suggested. However, a desmoid tumor needs to be excluded.

homogeneous low signal intensity equal to that of skeletal muscle on T1-weighted spin echo images, and equal to that of fat on T2-weighted images. The lesion contained central areas of markedly decreased signal intensity on T2-weighted images, reflecting areas of dense collagen within the lesion. The lesion also showed slight to moderate enhancement following administration of intravenous contrast medium. The mass lesion caused bulging of the right side of the abdominal wall and compressed the adjacent structures (bowel loops, right psoas muscle), extending medially and posteriorly, and reaching the right side of the fundus of the uterus. The appearance of this lesion was thought to represent a subserosal leiomyoma or an intra-abdominal fibromatosis, ie, a desmoid tumor.

The decision was made for laparotomy, which was performed via a midline incision by a skilled surgeon. A large mass attached firmly to the inner aspect of the abdominal wall was found intraoperatively (Figures $3 \mathrm{~A}$ and 3B). Surgeons took over and the mass was removed by wide excision with a safe margin (Figures 3C and 3D). The uterus was of normal size and completely free from the mass.

Histology reported a well circumscribed mass weighing $560 \mathrm{~g}$ and measuring $16 \times 11 \times 7 \mathrm{~cm}$, with a partially ruptured capsule. The outer surface was smooth with scanty muscle tissue seen in places. The cut surface was solid, whitish, fibrous, trabeculated, and glistening. No necrosis was seen. Microscopically, sections showed a circumscribed fibrous proliferation composed of fascicles of oval and spindle cells, with focal hyalinization and rich with thick-walled blood vessels. Spindle cells showed vesicular nuclei with 


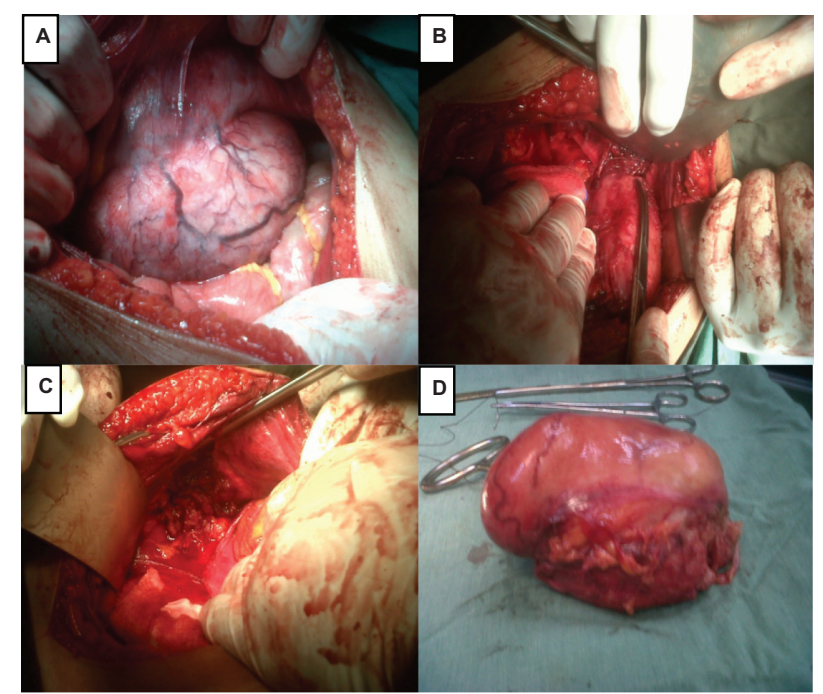

Figure 3 A) A picture showing the tumor in the inner aspect of the abdominal wall. B) The picture shows the margin of the sharp dissection. C) The tumor has been completely removed with a safe margin. D) The whole tumor after removal.

nucleoli, minimal atypia, and focal moderate mitosis. Neither necrosis nor nuclear anaplasia was seen. The diagnosis was an abdominal wall tumor consistent with desmoid-type abdominal wall fibromatosis.

\section{Discussion}

This case report shows that a large abdominal wall desmoid tumor on the inner aspect of the abdominal wall may mimic a large uterine subserosal leiomyoma. The case is interesting and unusual, because the imaging techniques used initially reported the possibility of a subserosal leiomyoma as the primary diagnosis rather than a desmoid tumor, because some sections in imaging had shown very close attachment of the tumor to the uterus. This case emphasizes the importance of clinical examination and a multidisciplinary approach to ensure the best outcome for the patient.

Some abdominal tumors may be clinically challenging for clinicians, especially those working in remote areas. The confusion in diagnosis may result from unusual locations, large-sized tumors, and discordant results obtained using different diagnostic tools. Determining tumor location and origin is more difficult when the tumor exceeds $5 \mathrm{~cm}$ in diameter. The majority of large pelvic masses in female patients represent commonly encountered entities, such as uterine fibroid tumors, dermoid tumors, ovarian cysts, and ovarian cancer. However, uncommon pelvic masses, such as mesothelioma, adenosarcoma, carcinosarcoma, leiomyosarcoma, and desmoid tumor, may also be encountered. Desmoid tumors may be located in the peritoneum, and can manifest as a large pelvic mass. Clinical symptoms are masked by slow growth of the tumor. Other clinicians have also reported misdiagnosis of desmoid tumors mimicking intra-abdominal tumors. ${ }^{11}$

In clinical practice, our first moments with a patient are filled with visual, auditory, and tactile information that determines both the effectiveness and cost of our subsequent care. One study had shown that $88 \%$ of diagnoses in primary care are established by the end of taking a brief history and physical examination. In another study, $56 \%$ of patients in a general medical clinic were assigned a correct diagnosis by the end of history-taking, and this figure rose to $73 \%$ by the end of their physical examination. ${ }^{12}$ However, despite the importance of the history and physical examination in the clinical setting, their accuracy and precision have rarely been subjected to rigorous evaluation. ${ }^{13}$

Many masses in the female pelvis arise from the reproductive organs. In addition, pelvic masses may arise from the gastrointestinal system, abdominal wall, urinary system, adjacent soft tissues, and metastases. Thus, the differential diagnosis for female pelvic masses is extensive. The site of origin (eg, uterus, cervix, adnexa, abdominal wall, rectum, bladder, pelvic muscles), clinical history and examination, and imaging findings may all help narrow the differential diagnosis. With large masses, it may not always be possible to determine the site of origin to differentiate between tumors at imaging. However, familiarity with the clinical examination and imaging features of common and uncommon pelvic masses is important for correct image interpretation. Finally, we must always remember that uncommon presentation of common disease is more common than the common presentation of rare diseases. ${ }^{14}$

Diagnostic tests are of crucial importance in clinical care and can help to determine the most appropriate treatment for individual patients, to monitor and modify ongoing treatments, and also to offer a prognosis. Furthermore, although we are all taught that a good history and physical examination have considerable value, many clinicians believe that there is little evidence to support the diagnostic utility of the clinical findings. ${ }^{13}$ Unfortunately, this belief has encouraged many clinicians, especially the younger ones, to reach for elaborate diagnostic tools before performing a thorough clinical examination as taught in medical schools and during fellowship programs, which has resulted in loss of the art of clinical examination.

\section{Disclosure}

The authors report no conflicts of interest in this work. 


\section{References}

1. Shields CJ, Winter DC, Kirwan WO, Redmond HP. Desmoid tumours. Eur J Surg Oncol. 2001;27(8):701-706.

2. Klemmer S, Pascoe L, DeCosse J. Occurrence of desmoids in patients with familial adenomatous polyposis of the colon. Am J Med Genet. 1987;28(2):385-392.

3. Acker JC, Bossen EH, Halperin EC. The management of desmoid tumors. Int J Radiat Oncol Biol Phys. 1993;26(5):851-858.

4. Adamian AA, Andreev SD, Annaev AG. Radical removal of desmoid with simultaneous plastic surgery of the defect in the anterior abdominal wall. Khirurgiia (Mosk).1989(5):123-124. Russian.

5. Bertani E, Chiappa A, Testori A, et al. Desmoid tumors of the anterior abdominal wall: Results from a monocentric surgical experience and review of the literature. Ann Surg Oncol. 2009;16(6):1642-1649.

6. Biermann JS. Desmoid tumors. Curr Treat Options Oncol. 2000; 1(3):262-266.

7. Abi Ezzi SS, Miller LS. The use of ultrasound for the diagnosis of soft-tissue masses in children. J Pediatr Orthop. 1995;15(5):566-573.
8. Bernathova M, Felfernig M, Rachbauer F, et al. Sonographic imaging of abdominal and extraabdominal desmoids. Ultraschall in der Medizin. 2008;29(5):515-519. German.

9. Szklaruk J, Tamm EP, Choi H, Varavithya V. MR imaging of common and uncommon large pelvic masses. Radiographics. 2003;23(2): 403-424.

10. Agostini S, Manara P. Desmoid tumor of the abdominal wall. Echographic and CT findings. J Radiol. 1985;66(8-9):531-533. French.

11. Ichikawa T, Koyama A, Fujimoto H, et al. Abdominal wall desmoid mimicking intra-abdominal mass: MR features. Magn Reson Imaging. 1994;12(3):541-544.

12. Sackett DL, Rennie D. The science of the art of the clinical examination. JAMA. 1992;267(19):2650-2652.

13. Straus SE, Mcalister FA, Sackett DL. Clinical assessment of the reliability of the examination (CARE). ACP J Club. 2000;133(2):A11-A12.

14. Smith CS, Pauww D. When you hear hoof beats: Four principles for separating zebras from horses. J Am Board Fam Pract. 2000;13(6): 424-429.

\section{Publish your work in this journal}

The International Journal of General Medicine is an international, peer-reviewed open-access journal that focuses on general and internal medicine, pathogenesis, epidemiology, diagnosis, monitoring and treatment protocols. The journal is characterized by the rapid reporting of reviews, original research and clinical studies across all disease areas.
A key focus is the elucidation of disease processes and management protocols resulting in improved outcomes for the patient.The manuscript management system is completely online and includes a very quick and fair peer-review system. Visit http://www.dovepress.com/ testimonials.php to read real quotes from published authors. 